\title{
DOES TOUCHING A PROJECTION AUGMENTED MODEL AND INTERACTING WITH IT USING A SPATIALLY- COINCIDENT DEVICE, AFFECT A USER'S PERCEPTION OF ITS SIZE?
}

\author{
Emily Bennett \\ Brett Stevens \\ Department of Information Systems and Computer Applications \\ University of Portsmouth \\ UK \\ emily.bennett@port.ac.uk
}

\section{ABSTRACT}

\begin{abstract}
A Projection Augmented model (PA model) is a novel type of display. It consists of a real physical model, onto which a computer image is projected to create a realistic looking object. PA models provide their users with whole-hand haptic feedback and support spatially-coincident haptic interaction devices. This paper reports on an experiment that investigated the effect these factors have on a user's perception of the size a PA model. Results showed that touching a PA model increased the accuracy of size estimates; however using a spatially-coincident haptic interaction device had no effect.
\end{abstract}

Keywords (Projection Augmented models, haptic, Tangible User Interface, Size Perception)

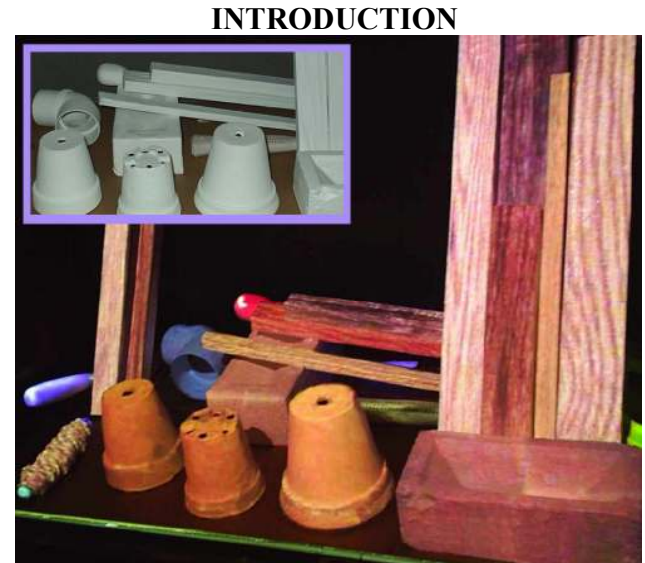

Figure 1: PARIS (main picture), PARIS without the projected image (inset)

A Projection Augmented model (PA model) is a type of projection based Augmented Reality display. It consists of a physical three-dimensional model, onto which a computer image is projected to create a realistic looking object (Stevens et al. 2001). For example, PARIS (Projection Augmented Reality Information System) consists of smooth white plaster models of objects commonly found in a garden shed. The image projected onto these objects provides colour and visual texture (figure 1).

The current paper reports on a user study that investigated what effect touching a PA model has on the perception of the size of its features. It is important to note when this paper refers to 'touch' or 
'haptic' it is actually referring to the situation when a PA model is being simultaneously viewed and touched. To put this study in context, it is first necessary to review the capabilities, applications and limitations of PA models.

\section{PROJECTION AUGMENTED MODELS}

To create a PA model, the image that is normally viewed on a PC monitor is projected directly onto a physical model. The projector and the physical model must be carefully aligned, so the projected image is correctly registered onto the physical model. Technology has been developed that semiautomates this process (Raskar et al. 2001), however it is possible to do this manually. Multiple projectors can be used to illuminate the PA model on all sides, and thus allowing the PA model to be viewed from any direction.

The projected image can change the perceived colour of the physical model. It can also make it appear to be made from a different material. For example, a dull clay vase can appear to be made out of a shiny ceramic material (Raskar et al. 2001). Different lighting conditions can also be simulated (Raskar et al. 2001). Additionally, by projecting an animated image onto the static physical model, apparent motion can be simulated (Raskar, Ziegler \& Willwacher 2002). Text or other information can be added to the projected image. For example, the image projected onto a PA model of a fossil can include annotations (Bimber et al. 2002). Examples of applications for which PA models are currently being developed include product design (Verlinden et al. 2004), geographical visualization (Piper, Ratii, \& Ishii 2002) (Clark et al. 1998) and museum exhibits (Exhibition at the Museum of London 2004) (Bimber et al. 2002).

PA models have several advantages over other types of displays. A PA model gives a threedimensional physical shape to a two-dimensional computer image. People can touch the surface of a PA model with their bare hands. Therefore PA models provide whole-hand haptic feedback, without the need to wear any additional equipment.

Additionally, multiple people can simultaneously view a PA model. Each person has their own unique view, which is dependant on their physical location. This is because PA models are real physical objects therefore each person positioned around it, perceives it from a different direction. For example, people seated on the left side of PARIS (figure 1) would see the left side of the objects (e.g. plant pots and bricks), whereas someone standing up and looking down would see the top of the objects. This can be contrasted with other displays such as a standard monitor, which present an image from a single viewpoint, and everyone sees the same image regardless of their location. Some displays can support multiple users, with each user having their own unique position-dependant viewpoint. However, the number of users these displays can support is limited by the current technology, whereas the number of multiple users that a PA model can support is only limited by the physical space around the model.

Despite the potential benefits and applications of PA models, they have some limitations. These limitations highlight the ways in which PA models may differ from their real life counterparts. The most notable limitation is that PA models normally have a fixed physical shape. Although deformable PA models have been built e.g. (Piper, Ratii, \& Ishii 2002) (Iwata et al 2001), they can only change shape within a limited range.

A second limitation is that there is a perceptual mismatch between visual and haptic feedback for some properties of a PA model. For example, a PA model cannot simulate properties such as physical texture. This is because to enable it to be possible to project onto a PA model, the physical model must have a smooth diffuse surface. Therefore when a person touches a PA model, its visual texture (from the projected image) does not match its physical texture. For example, when a person touches a PA model that looks like it is made from metal, they realize it does not feel like metal (Raskar et al. 2001). However, it is worth pointing out that this limitation is only relevant when a PA AJIS Special Issue 
model represents an object on a real life scale. This is much less likely to be an issue for PA models that represent objects on a different scale to real life.

A further problem is that when a person touches a PA model, the projected image appears on the back of their hand, and their hand casts a shadow onto the PA model. Multiple projectors and computer vision techniques can reduce this problem (Mukaigawa, Nagai \& Ohta 2004). This problem can be overcome completely by using a back-projected PA model e.g. (Clark et al. 1998).

\section{RESEARCH PROJECT AND RESEARCH QUESTION}

The research presented in this paper is part of a larger project. To put this paper in context, it is important to outline the aims and objectives of this project.

\section{Project overview}

The research into PA models has focused on developing the enabling technology; very few studies into how people use PA models have been conducted. It is important to carry out user studies on a new type of display early in its development (Lampton, Bliss \& Morris 2002). When conducting human centred studies with new displays that do not have a main application area, it is advisable to evaluate them in terms of 'basic' tasks, such as perception and memory (Hale and Stanney 2004 ; Kirkpatrick \& Douglas 2002). A main application area for PA models has not yet emerged; therefore this project will focus on 'basic' tasks to allow guidelines to be created that can be generalised to many applications.

A review of PA models has shown that they provide extensive haptic feedback because the user can touch them with their bare hands. However, it has been noted that touching a PA model may actually reduce its realism. The feedback provided by PA models is of particular interest because it could both benefit and hinder the user, depending on the task they are performing. PA models should be considered as part of a user interface system, any user interface system needs both a display and an interaction device (Latta \& Oberg 1994). Therefore both haptic feedback and haptic interaction are important and they should be studied together.

The aim of this research project is to create guidelines that will enable a person who is interested in using a PA model for a particular application, determine the best way of making use of the haptic feedback and the haptic interaction devices that PA models can support, to meet the requirements of that application. These guidelines will indicate which tasks benefit from touching and haptically interacting with a PA model. They will also suggest the factors for which touch and haptic interaction may have a detrimental effect. 


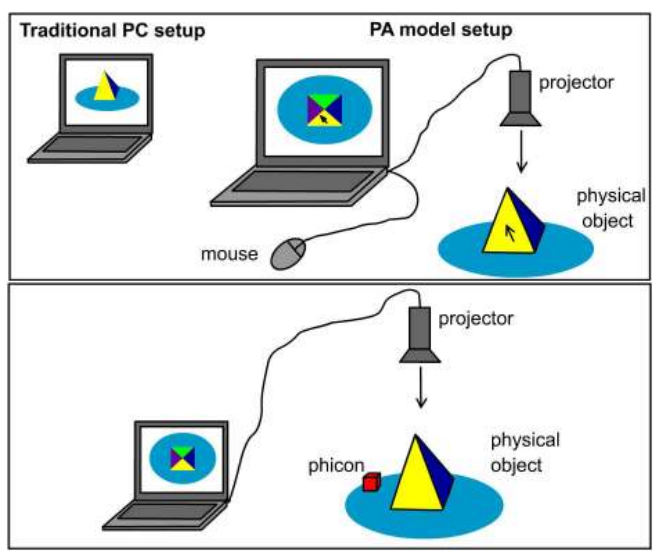

Figure 2a: Interacting with a PA model using a mouse, Figure 2b: Interacting with a PA model using a TUI

To clarify the aims of this project further; the term 'haptically interacting' refers to interaction devices that enable the user to perceive the haptic properties of a PA model. Such devices can be referred to as spatially-coincident devices. An example of a spatially-coincident device is a Tangible User Interface (TUI). A TUI allows a person to use small physical objects to interact with digital information (Ullmer \& Ishii, 2001). These physical objects are referred to as phicons (physical icons). A TUI can be used to interact with a PA model by allowing the user to place a phicon directly on its surface (figure 2b). Spatially-coincident interaction devices can be contrasted with spatially-separate devices. When using a spatially-separate device, the action performed by the user is in a different location from the PA model, thus the PA model is not haptically perceived. An example of a spatially-separate device is a mouse. When using a mouse, the user moves it in a separate location to the mouse pointer, which is projected onto the physical model (figure 2a).

\section{Aims of the first study and scope of this paper}

As part of this project, the first study was conducted to investigate the effect that touching a PA model and interacting with it using a spatially-coincident device, has on users' perception of its size and their sense of object-presence. Object-Presence is the feeling that an object exists in a person's environment, when that object does not (Stevens et al 2001). This paper focuses on the size perception results.

It is important to note that object-presence was investigated using a post-test questionnaire, and a between-participants experimental design was used. Therefore the measurement of object-presence could not have affected the size perception results described in this paper.

\section{HYPOTHESES}

The research question this paper investigates is: what effect does touching a PA model and interacting with it using a spatially-coincident device, have on users' perception of its size? Separate hypotheses can be made about the effect of touching and the effect of using a spatially-coincident interaction device. 


\section{Hypothesis 1}

The first issue this study investigates is concerned with the effect touching a PA model has on the accuracy of the users' size estimates. It has been shown that vision is the dominant sense for perceiving size (Ernst \& Bülthoff 2004). This suggests that touching a PA model will not increase the accuracy of the perception of its size. However, there is some conflicting evidence, for example it has been shown kinesthetic feedback dominates over vision for the perception of depth (Beers, Woolpert \& Haggard 2002). Moreover, it has been demonstrated that perception is more accurate when both visual and haptic feedback are combined.

Several models have been proposed which all suggest that more accurate perception can be achieved by combining the senses, see (Ernst \& Bülthoff 2004) for a review. For example, the Optimal Integration Model predicts that the way in which humans combine sensory information depends on the situation, and in some cases more accurate perception can be achieved by using multiples senses (Ernst \& Banks, 2002). This model is supported by empirical research that found the addition of haptic feedback to visual feedback increased the accuracy of size estimates (Gepshtein \& Banks, 2003). Therefore it is hypothesized that the accuracy of size estimates will be higher when a PA model simultaneously viewed and touched, compared to when it is just looked at.

It is important to note that it may appear that this hypothesis has already been tested, however the visual feedback provided in Gepshtein et al's (2003) experiment only contained the visual depth cue of stereopsis, it did not contain other depth cues such as texture gradient. It is possible that if more depth cues were provided, the haptic feedback may not have had such an effect on perception because the visual information would have been sufficient for the participants to accurately perceive size. Given that a PA model provides feedback for all visual depth cues, Gepshtein et al (2003) does not provide conclusive evidence that touching a PA model will increase the accuracy of perception. Indeed, it has been argued that it is important that research into multi-sensory integration and perception (such as this study), should be ecologically valid (De Gelder \& Bertelson 2003). Therefore it is necessary to conduct empirical research with actual PA models, opposed to relying on previous work.

\section{Hypothesis 2}

The second issue that this study is investigating is concerned with the effect that interacting with a PA model using a spatially-coincident interaction device has on the accuracy of the users' size estimates. To operationalise this question, the two interaction devices that this study focuses on are a mouse (spatially-separate) and a TUI (spatially-coincident) (see section 3.1 for details). Spatiallycoincident interaction devices such as TUIs, require the user to physically touch the surface of the PA model. This means that the users haptically perceive the PA model's physical shape, while they are interacting with it. Thus spatially-coincident devices such as TUIs, provide kinesthetic (limb position) feedback and single-point force feedback. This may have an effect on how accurately the users' can estimate the size features of a PA model.

The decision was made to investigate a priming effect because it more accurately reflects how user interfaces are used in real life. In real life, a user may be asked for information (e.g. a size estimate) about the display that they have been interacting with. In making this estimate, they may implicitly use information they gained previously while they were interacting with the display. However, it is unlikely they would actually use the interaction device to make their estimate. Therefore this hypothesis is concerned with size estimates made a short time after using a particular device. (Opposed to the first question in section 4.1 that is concerned with size estimates made almost simultaneously to the visual or haptic feedback being perceived). 
The same research into the effect of combining visual and haptic feedback, which lead to the first hypothesis (section 4.1), is also relevant to this question. This research suggests that using a TUI is likely to result in people making more accurate size estimates. This is supported by empirical evidence that is directly relevant to this question. It has been demonstrated that combining visual and kinesthetic feedback results in a more accurate estimate of the position of a finger, compared to using visual feedback alone (Beers, Woolpert \& Haggard 2002) (Beers, Stittig \& Denier 1996). This suggests that the kinesthetic feedback gained from using a TUI will enable people to made more accurate size estimates, compared to if they used a mouse that does not provide kinesthetic feedback. However, caution must be taken in applying previous research to this study. This study is concerned with size estimates that are made a short time after the interaction has taken place. However, the research into the effect of combining sensory modalities is concerned with measures taken whist or directly after the stimulus is perceived. Therefore memory is also a factor that needs to be considered.

According to the Information Processing Approach, the perceptual system and memory are linked, which suggests that if a stimulus is more accurately perceived, then it will be more accurately remembered (Eysneck \& Kean 2001). This indicates that previous research which demonstrated combining modalities results in more accurate perception, also indicates that memory will be more accurate. Therefore it is hypothesized that the accuracy of size estimates will be higher after a TUI is used, compared to after a mouse is used.

\section{EMPIRICAL STUDY}

\section{Method}

A total of 80 participants (55 male 25 female) were used. They were all students on computingrelated degree courses. The mean ages were 24 for males and 20 for females. An equal number of male and females were used in each condition (+/-1).

PARIS (Projection Augmented Reality Information System) (figure 1) is the PA model that was constructed for this experiment. This PA model consisted of white plaster models of various objects that are commonly found in a garden shed. The image projected onto these objects gives them colour and visual texture.

PARIS represents objects that are likely to be familiar to the participants. Although this was necessary for the part of the study that investigated object-presence (not reported in this paper), it is a potential problem for this part of the study. Participants' size estimates may be affected by their previous knowledge. However, this is unlikely to be an issue because apart from the bricks, none of the objects in PARIS have a standard size in real life. Moreover, most of the size estimates were of distances between objects or of parts of objects.

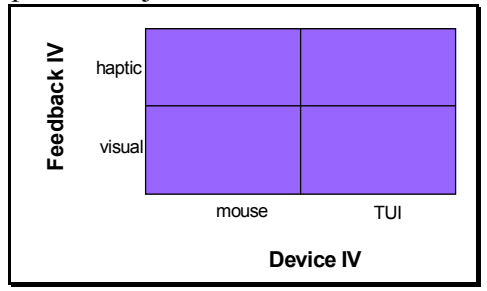

Figure 3: Experimental Conditions 
A $2 \times 2$ between-participants factorial design was used (figure 3). There were two IVs; Device and Feedback (figure 3).
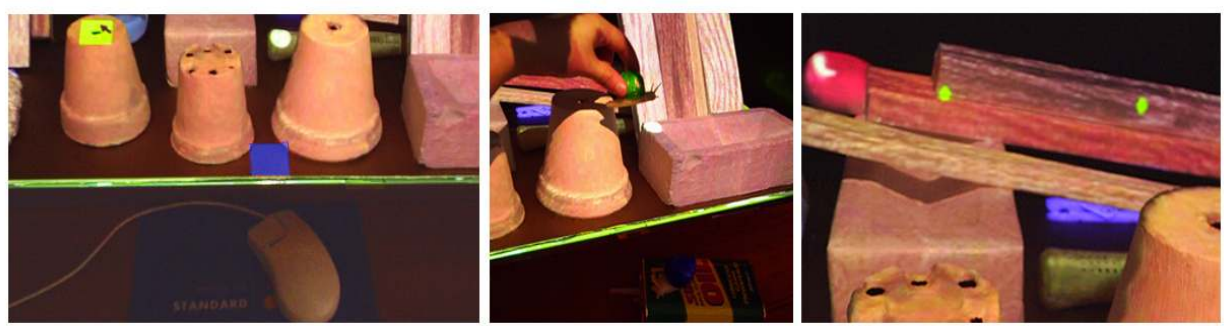

Figure 4a: Mouse condition, Figure 4b: TUI condition, Figure 4c: Size estimate markers

The Device IV was split into two levels of Mouse and TUI. The device conditions were to prime the participant with either using a TUI or a Mouse (hypothesis 2). The participants were required to complete a manipulation and target acquisition task. In the Mouse conditions this involved the manipulation of a projected green square to select various parts of the PA model (figure 4a). In the TUI conditions they had to use a phicon (plastic snail) to select various parts of the PA model (figure $4 b)$.

The Feedback IV was split into two levels of Visual and Haptic. Participants in both conditions were seated in front of the apparatus. They were asked to make size estimates of various features of PARIS. Pairs of markers were projected onto PARIS (figure 4c). The participants had to give an estimate of the distance between them (from their furthest most points). Participants in the Visual condition made their estimate by just looking at the markers. Participants in the Haptic condition made their estimate by both looking at and touching the markers. Precisely how the participants gave their size estimates is explained in section 5.11. The procedure of the experiment is as follows:-

1. Participants completed the priming task by interacting with PARIS using either a TUI or the mouse (Device IV).

2. Participants then made size estimates of various parts of PARIS. They did this by either looking at it, or by both looking at it and touching it (Feedback IV). Their size estimates were recorded.

3. Finally, participants completed object-presence questionnaires (not reported in this paper).

\section{Measures}

Certain restrictions on how participants made their size estimates were necessary to ensure the experiment was valid. The participants in the Haptic conditions were told to touch the two dots with their thumb and index finger. If the distance between the dots was too great, they were told to use the index fingers on both hands. This created a slight problem because the participants all had different size hands. Hence, there were some sizes which some participants made with one hand and others which they made with both hands. This problem was over come by using range of sizes, which meant all participants made some judgments with one hand and some with both hands. Once they had touched the markers, they had to place their hand flat down on the table, to ensure they lost the position of their fingers.

AJIS Special Issue

December 2004 
A further restriction was imposed on the participants in the Haptic conditions. They were not allowed to touch the markers and hold their fingers in a fixed position, and then rotate their hand to get a better view of the distance between their fingers. If they did this, they would actually be making judgments based on visual feedback. It could be argued that this restriction is not ecologically valid because in real life people would be free to do this. However, by allowing people to do this, it would mean the experiment would really be investigating the effect of changing the viewpoint, as opposed to the effect of haptic feedback.

This leads to a further restriction that was imposed in both the Haptic and the Visual conditions. Participants were instructed not to move forward or change their angle of view when making the estimates. This instruction aimed to ensure that everyone perceived all of the sizes from roughly the same viewpoint, thus minimize the difference in the visual feedback received by participants.

The decision of how participants should actually give their size estimates was problematic. It has been argued that when comparing visual and haptic senses, the results can be affected by the method used to record the participants' response (Welch \& Warren, 1980). It is therefore necessary to explain the measures used in this experiment.

Perception of size can be measured using either relative or definite estimates. The procedures required and the type of data generated from relative estimate tasks, were not suitable for this experiment (Bingham \& Pagano 1998).

Definite estimates can be measured using either verbal or action estimates. Verbal estimates involve giving a verbal estimate in specific units, for example in centimetres. However, it has been found that verbal estimates vary considerably from person to person (Bingham et al., 1998). This problem can be overcome to some extent, by placing a calibrating device such as a ruler near the object that is being perceived (Bingham et al., 1998). However, it has been shown that visual feedback dominates when this method is used (Heller, Calcaterra, Green \& Brown, 1999). This suggests that this method is not suitable when studying multi-sensory perception because it may not be sensitive enough to pick up information gained through the haptic sense. Therefore a verbal estimates measure was not used.

Action estimates are the other type of definite judgment that can be used to measure the perception of size. An action estimate typically requires the participant to view an object, and then give a size estimate by making an action, such as reaching out an arm (Pagano, Grutzmacher \& Jenkins, 2001). Importantly, the participant cannot see their hand or arm while making their estimate. It has been argued that action estimates are a more valid way of measuring perception than verbal estimates (see Bingham et al., 1998 for a review). However, it has been found that the haptic sense dominates when this method is used (Heller et al., 1999). This suggests that this type of measure may be more sensitive to haptic feedback than to visual feedback. Therefore, if this method was used and size was more accurately estimated in the Haptic conditions, it may actually be because the method was very sensitive to haptically perceived information.

An alternative to this type of action estimate is to allow participants to see their hands while they are making their judgement. However, this brings the opposite problem to the one described above. It has been argued the reason that many studies have shown vision to be the dominant sense in the perception of size, is because this type of method was used (Heller \& Schiff, 1991). It has been suggested that this type of method is not sensitive enough to detect haptically perceived information (Heller et al., 1991). Therefore, if this method was used and there was no difference in the accuracy of the size estimates between the Haptic and Visual conditions, this may actually be because the method is not sensitive enough to detect haptically perceived information.

Given these problems with both types of action estimate, if either estimates were used alone, it would not be possible to gain a full and accurate picture of the effect IVs may have had. Therefore two different action estimates of size were used; one where the participant could not see their hands (Nosight), and another where they were able to see their hands (Sight). The participants were required 
to make a pincer movement with their fingers to indicate their size estimate. The experimenter recorded the distance between their fingers. A total of 20 different sizes were used (20 pairs of markers). For each pair of markers the participant gave a Sight and a No-sight estimate, thus two sets of data were obtained.

The markers were displayed while the participants gave their estimate. Roughly an equal number of height, width and depth estimates were used. If the size was too big, they were instructed to use both hands when giving their estimates. Importantly, participants in the Haptic conditions were instructed to use the same hand when giving their estimate, as they used when perceiving the size. This is because there is no automatic perceptual connection between the hands (Lange, Klatzky \& Ernst, 2004).

\section{Results}

Two sets of size estimates were obtained; Sight estimates and No-sight estimates (section 5.11). For both data sets, the accuracy of the participants' estimate for each distance was calculated by taking away their estimate from the correct distance. Thus, a positive number indicated an overestimate and a negative number indicated an underestimate.

From both the Sight and No-sight data sets two values will be investigated; an Absolute value and a Signed value. The Absolute for each participant is the mean of his or her 20 accuracy values, after turning all underestimates into positive numbers. This gives an overall indication of how accurate a person was. Thus higher values indicate a higher level of error in their estimates. A very brief summary of the Absolute values results has been published previously (Bennett \& Stevens 2004).

The Signed value for each participant is the mean of his or her 20 accuracy values, when the signs of the estimates are not changed. This gives an indication of the direction of the error. Positive Signed-values indicate on average the participant is making overestimates, and negative Signedvalues indicate on average the participant is making underestimates.

The results from the two data sets are presented separately; the Sight estimate results are presented first (both Absolute and Signed values), then the No-sight estimate results (both Absolute and Signed values).

Sight Estimate
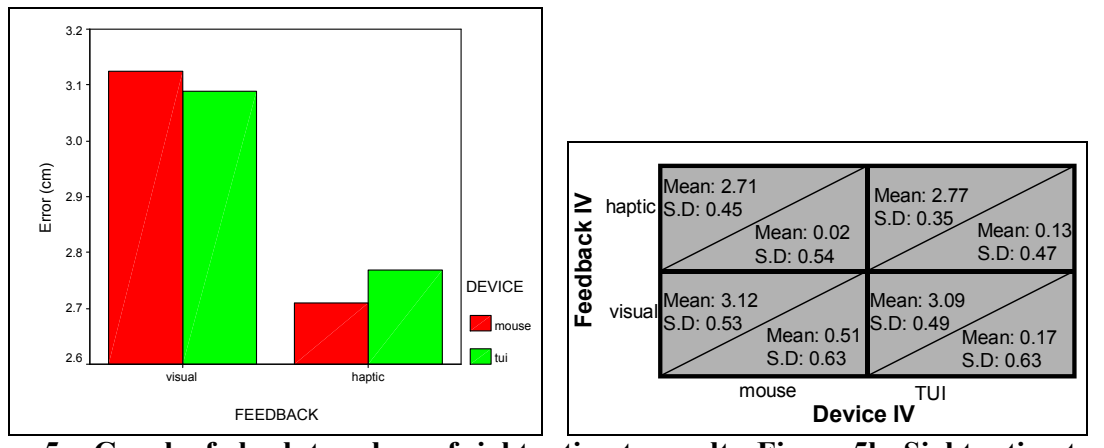

Figure 5a: Graph of absolute values of sight estimate results, Figure 5b: Sight estimate results - absolute values above diagonal, signed values below diagonal

The Absolute values and the Signed values for the Sight estimates were analysed separately, using a two-way between-participants ANOVA. Analysing the Absolute values (figure 5b) found a 
significant effect for Feedback $(\mathrm{F}(1,76)=12.92, \mathrm{p}<0.001)$, no significant effect for Device $(\mathrm{F}(1,76)=0.02, \mathrm{p}=0.90)$ and no significant interaction $(\mathrm{F}(1,76)=0.22, \mathrm{p}=0.64)$. This effect is illustrated in the graph in figure 5a. Analysing the Signed values (figure $5 \mathrm{~b}$ ) found a significant effect for Feedback $(F(1,76)=4.26, p<0.05)$, but no significant effect for Device $(F(1,76)=0.87$, $\mathrm{p}=0.35)$, and no significant interaction $(\mathrm{F}(1,76)=3.20, \mathrm{p}=0.08)$.

No-sight estimate


Figure 6a: Graph of absolute values of no-sight estimate results, Figure 6b: No-sight estimate results - absolute values above diagonal, signed values below diagonal

The Absolute values and the Signed values for the No-sight estimates were analysed separately, using a two-way between-participants ANOVA. Analysing the results from the Absolute values (figure $6 \mathrm{~b}$ ) found a significant effect for Feedback $(\mathrm{F}(1,76)=34.38, \mathrm{p}<0.001)$, no significant effect for Device $(F(1,76)=3.73, p=0.06)$ and no significant interaction $(F(1,76)=1.54, p=0.22)$. This effect is illustrated in the graph in figure 6a. Analysing the results from Signed values $(6 \mathrm{~b})$ found no significant effect for Feedback $(\mathrm{F}(1,76)=0.21, \mathrm{p}=0.65)$, no significant effect for Device $(\mathrm{F}(1,76)=0.33, \mathrm{p}=0.57)$, and no significant interaction $(\mathrm{F}(1,76)=0.01, \mathrm{p}=0.97)$.

\section{Discussion}

The discussion first focuses on the Absolute values for both the Sight and the No-sight estimates because it is the Absolute values that determine if the hypotheses are supported. It then examines the Signed values for both the Sight and No-sight estimates.

For both the Sight (section 5.2.1) and No-sight estimates (5.2.2), it was found that the Absolute values of the estimates that were made with haptic feedback, were significantly lower than those made with only visual feedback. This supports the hypothesis that size is more accurately estimated when the PA model is touched, compared with when it is just looked at (section 4.1).

For both the Sight (section 5.2.1) and the No-sight estimates (section 5.2.2), there was no significant difference between the absolute values of the estimates made after using a TUI, compared to those made using a mouse. Therefore the hypothesis that the accuracy of size estimates will be higher after a TUI is used, compared to after a mouse is used, is not supported.

This can be explained by previous research. It has been found that combining visual and haptic feedback results in more accurate perception of size (section 4.1). However, there is also a large body of research that has shown the visual sense dominates during perception of size (Ernst \& Bülthoff 2004). This suggests that the effect haptic feedback has on perception may depend on the type of haptic feedback that is being provided. Thus the single point-of-contact haptic feedback 
provided by a TUI, may not be sufficient to increase the accuracy of the perception of size. This prediction is supported by research that found haptic feedback perceived through a single point is not sufficient for a person to accurately perceive the size and shape of an object (Klatzky \& Lederman 2004). This has also been shown to be true for single-point-of-contact haptic devices, such as a PHANToM (Kirkpatrick \& Douglas 2002).

Memory may also have been a factor that lead to no significant difference being found between the TUI and the Mouse conditions. The size estimates were made a short time after the participants completed the priming (interaction) task. It is possible that the haptic feedback gained in the TUI conditions, was lost due to the time delay.

The results can be investigated in more depth by looking at the results for the Signed values. The Signed values indicate the direction (overestimate or underestimate) of the results in each condition. For both the Sight and the No-sight data sets, in all four conditions the Signed values are positive, therefore in all conditions on average participants slightly over estimated sizes. When the No-sight estimate was analysed, there was no significant difference between signed values. This indicates that for this measure, neither IV has an effect on the direction of the estimates. However, when the Sight estimates were analysed, there was a significant effect for Feedback, with lower overestimates being made in the Haptic conditions. This suggests that for the Sight estimates, haptic feedback can reduce the tendency to overestimate sizes. Taking the pattern of the results for the No-sight and the Sight estimates together; they suggest that people can be affected by the sight of their hands when making size estimates. A similar pattern has been found in previous research (Heller et al. 1999).

Returning to the original research question that this study is investigating. I.e. What effect does touching a PA model and interacting with it using a spatially-coincident device, have on users' perception of its size, when compared to not touching it and using a spatially-separate device? (section 3.2) The effect of touching a PA model has been tested directly (see above). The effect of using a spatially-coincident device or spatially-separate device was operationalised using a TUI and a mouse. Therefore these results suggest that using a spatially-coincident device does not affect the users' perception of size, compared to using a spatially-separate device.

The question investigated in this study is part of a larger project. The projected aims to suggest a number of guidelines that will enable a person who is interested in using a PA model for a particular application, determine the best way of making use of the haptic feedback that PA models can support, to meet the requirements of that application (section 3.1). The guideline that can be derived from these results is; for applications for which size perception is important, the users should be instructed to touch the PA model with their bare hands, and it is not important whether a spatiallycoincident or spatially-separate device is used. It is important to note that these are preliminary guidelines; more work is needed to determine how touch affects other factors.

\section{CONCLUSIONS}

This study found that size estimates made when a PA model is simultaneously viewed and touched are significantly more accurate than those made when it is just looked at. This study also found that there was no significant difference in the accuracy of estimates of size made after interacting with a PA model using a spatially-coincident device, compared with after using a spatially-separate device. This research is part of a larger project that aims to suggest a number of guidelines that will enable a person who is interested in using a PA model for a particular application, determine the best way of making use of the haptic feedback that PA models support, to meet the requirements of that application. These results can be used to contribute to the formation of these guidelines.

The findings from this study are also related to the wider area of haptic perception and haptic displays. They support previous work, which found haptic feedback increases the accuracy of the 
perception of size. The results of this study also support previous work that found single-point haptic feedback is insufficient in the perception of size.

\section{REFERENCES}

Bennett, E. and Stevens, B. (2004). The Effect that Haptically Perceiving a Projection Augmented Model the perception of size, (poster). In Proceedings of International Symposium of Mixed and Augmented Reality (ISMAR04). In press.

Beers, R., Woolpert D. and Haggard, P. (2002) When Feeling Is More Important Than Seeing in Sensorimotor Adaptation. Current Biology, Elsevier, 12, 834-837.

Beers, R., Stittig, A. and Denier, J. (1996). How humans combine simultaneous proprioceptive and visual position information. Experimental Brain Research, Springer, 111, 253-261.

Bimber, O., Gatesy, S., Witmer, L., Raskar, R. and Encarnacao, L. (2002) Merging Fossil Specimens with Computer-Generated Information. IEEE Computer. 35(9), 25-30.

Bingham G. and Pagano, C. (1998) The Necessity of a Perception-Action Approach to Definite Distance Perception: Monocular Distance Perception to Guide Reaching. Journal of Experimental Psychology, 1998, 24(1), 145-168.

Clark, D. McKeon, R., Marciano, R. and Bailey, M. (1998) Rear-Projecting Virtual Data onto Physical Terrain: An Exercise in Two Senses Being Better Than One. In Proceedings of IEEE Visualization, IEEE Press, 451-454.

Ernst, M and Banks, M. (2002) Humans integrate visual and haptic information in a statistically optimal fashion. Nature, 415, 429-433.

Ernst, M and Bülthoff, H. (2004) Merging the senses into a robust percept. Trends in Cognitive Sciences, Elsevier, 8(4), 162-169

Eysneck, M and Kean, M Cognitive Psychology, $\left(4^{\text {th }}\right.$ Edition). 2001, Psychology Press.

Exhibition at the Museum of London. Available online at: http://www.museumoflondon.org.uk/MOLsite/exhibits/bodies/bath.htm, Accessed May 2004.

De Gelder, B and Bertelson, P (2003) Multisensory integration, perception and ecological validity. Trends in cognitive science, Elsevier, 7(10), 460-467.

Gepshtein, S. and Banks, M. (2003) Viewing Geometry Determines How Vision and Haptics Combine in Size Perception, Current Biology, Elsevier, 13(6), 483-488, 2003

Hale K. and Stanney, K. (2004) Deriving Haptic Design Guidelines from Human Physiological, Psychophysical, and Neurological Foundations. IEEE Computer Graphics and Applications. 24(2), 33-39.

Heller, M. Calcaterra, J. Green, S and Brown, L (1999) Intersensory conflict between vision and touch: The response modality dominates when precise action-riveting judgments are required. Perception and Psychophysics, 61(7), 1384-1398.

Heller, M. and Schiff, W. (1991) Psychology of touch. Lawrence Erlbaum Associates New Jersey, USA.

Iwata, H., Yano, F. Nakaizumi, and Kawamura, R. (2001) Project FEELEX: Adding Haptic Surface to Graphics. In Proceedings of International conference of computer graphics and interactive techniques (SIGGRAPH '01), ACM Press, 469-476.

Kirkpatrick, A. and Douglas, S. (2002) Application-based evaluation of haptic interfaces. In Proceedings of 10th Symposium on Haptic Interfaces for Virtual Environment and Teleoperator Systems, IEEE Computer Society Press, 32-40.

Klatzky, R. and Lederman, S. (2003) Touch. In A. Healy and R. Proctor (eds.) Handbook of Psychology, volume 4: Experimental Psychology. Wiley, New York. 
Lampton, D., Bliss, J. and Morris, C. (2002) Human performance measurements in virtual environments. In K. M. Stanney (Eds.) Handbook of Virtual Environments: Design, Implementation, and Applications, Lawrence Erlbaum Associates, New Jersey, USA.

Lange, C., Klatzky, R. and Ernst, M. (2004) Bimanual Size Estimation: No Automatic Integration of Information across the Hands. (in press) available online: http://www.kyb.mpg.de/publications/pdfs/pdf2722.pdf Accessed June 2004.

Latta, J. N. and Oberg, D. (1994) Conceptual virtual reality model. IEEE Computer Graphics and Applications, 14(1), 23-29.

Mukaigawa, Y., Nagai, H. and Ohta, Y. (2004) Space Relighter: Technical report of the Institute of Electronics, Infomation and Communication Engineers. www.image.esys.tsukuba.ac.jp/ mukaigaw/papers/PRMU2004-SpaceRelighter.pdf, Accessed June 2004

Pagano, C., Grutzmacher, R. and Jenkins, J. (2001) Comparing Verbal and Reaching Responses to Visually Percieved Egocentric Distances. Ecological Psychology, Lawrence Erlbaum Associates, 13(3), 197-226.

Piper, B., Ratii, C. and Ishii, H. (2002) Illuminating clay: a Tangible Interface with potential GRASS applications. In Proceedings of Open Source Geographical Information Systems - GRASS users conference.

Raskar, R., Welch, G., Low K. and Bandyopadhyay, D. (2001) Shader Lamps: Animating Real Objects With Image-Based Illuminations. In Proceedings of the $12^{\text {th }}$ Eurographics Workshop on Rendering, Springer.

Raskar, R. Ziegler R. and Willwacher, T. (2002) Cartoon Dioramas in Motion. In Proceedings of International Symposium on Non-Photorealistic Animation and Rendering (NPAR), ACM Press.

Stevens, B., Jerrams-Smith, J., Heathcote, D. and Callear, D. (2002) Putting the Virtual into Reality: Assessing Object-Presence with Projection-Augmented Models. Presence: Teleoperators and Virtual Environments, MIT Press, 11(1), 79-92.

Ullmer, B and Ishii, H. Emerging frameworks for tangible user interfaces. Carroll, J. M. HumanComputer Interaction in the New Millennium. Boston, USA: Addison-Wesley Professional; 2001; pp. 579-601

Verlinden, J., Esker, W., Wind, L. and Horváth, I. (2004) Qualitative comparison of virtual and augmented prototyping in handheld products. In Proceedings of International Design Conference - Design 2004, Dubrovnik.

Welch R. and Warren, D. (1980) Immediate Perceptual Response to Intersensory Discrepancy, Psychological Bulletin, 88(3), 638-667.

\section{COPYRIGHT}

Emily Bennett and Brett Stevens (C) 2004. The authors assign to OZCHI and educational and nonprofit institutions a non-exclusive licence to use this document for personal use and in courses of instruction provided that the article is used in full and this copyright statement is reproduced. The authors also grant a non-exclusive licence to OZCHI to publish this document in full in the Conference Papers and Proceedings. Those documents may be published on the World Wide Web, CD-ROM, in printed form, and on mirror sites on the World Wide Web. Any other usage is prohibited without the express permission of the authors. 